\title{
Be aware, make it clear, and take the Lead: emotion regulation difficulties and emotional intelligence as moderators of cognitive reappraisal
}

\author{
Barbara Bodrogi $^{1} \cdot$ Tamas Bereczkei $^{1} \cdot$ Anita Deak $^{1}$ (D) \\ Accepted: 11 November 2020 / Published online: 17 November 2020 \\ (C) The Author(s) 2020
}

\begin{abstract}
Two research traditions explain the way we deal with emotional situations: emotional intelligence (EI) and emotion regulation (ER). EI refers to the individual differences in the knowledge, identification, and regulation of emotions. ER describes processes in which emotions are experienced, expressed, and altered. Our study examined the EI-ER link and their moderating role on affective responses. We used self-report questionnaires and a cognitive reappraisal (CR) task, in which subjective affective responses were registered. We found that higher levels of ER difficulties correlated with lower EI. Gender had an overall impact on affective changes, indicating a more unpleasant and more arousing affective state for women compared with men. Regarding the moderating role of EI and ER difficulties, the ability to utilize emotions (Utilization) decreased the valence into a more unpleasant direction, similar to the effect of the inability to identify and differentiate emotions (Clarity). A weak control over emotions (Impulse), however, increased the valence into a more pleasant direction. The lack of attention to emotional signals (Awareness) marginally decreased the initial intensity (i.e., lower level of arousal). We demonstrated that EI and ER have distinctive routes and a different influence on the affective outcome defined by valence and arousal ratings: (1) EI has an impact through the utilization of emotions mainly on the valence dimension; and (2) individual differences in ER have a moderating effect on both valence and arousal dimensions. This study provided evidence on how individual differences contribute to a successful ER process when using a CR strategy.
\end{abstract}

Keywords Emotional intelligence $\cdot$ Emotion regulation $\cdot$ Valence $\cdot$ Arousal $\cdot$ IAPS

Although great scientific interest has been directed toward the cognition-emotion interaction, little is known about the overlap between emotion regulation (ER) skills and other constructs containing an emotion regulatory and/or managing component such as emotional intelligence (EI). In this paper, we uniquely combine the tradition of ER (Gross, 1998, 2015) and the tradition of the individual differences in identifying, understanding, expressing, and regulating emotions through the concept of EI.

EI has been a controversial psychological construct and questions have arisen relating to its concept and future. Antonakis and colleagues (Antonakis, Ashkanasy, \& Dasborough, 2009; Antonakis \& Dietz, 2010) drew attention to the significance of empirical support and consensus in

Anita Deak

deak.anita@pte.hu

1 Institute of Psychology, University of Pecs, Ifjusag Str. 6., Pecs H-7624, Hungary measurements of EI. They identified many problems with ability and especially trait EI tests and suggested that more precise and specific definition(s) would help the development of the field. Consequently, one of their proposals is to "commit firmly to the ability definition" (Antonakis \& Dietz, 2010, p. 8) and another is to use objective designs such as physiological, biological measures.

With the growing number of studies, more and more links have been found to cognitive processing. The three-branch model contains factors such as Perception, Understanding, and Management (Fan, Jackson, Yang, Tang, \& Zhang, 2010). The tripartite model of EI (Mikolajczak, Petrides, Coumans, \& Luminet, 2009) differentiates three levels: knowledge, abilities, and traits. Knowledge means what individuals know about emotions. Abilities determine whether they can apply this knowledge (e.g., Are they able to express their emotions?). Traits are identified as emotion-related dispositions, in other words how individuals typically behave in a certain emotional situation. Recently, EI has been considered a type of cognitive ability and structured under the Cattell- 
Horn-Carroll (CHC) model of intelligence as a secondstratum factor of intelligence that appears in the emotional domain (MacCann, Joseph, Newman, \& Roberts, 2014).

Based on the early three-branch EI model of Salovey and Mayer (1990), in which the three main dimensions are (1) the appraisal and expression of emotion, (2) the regulation of emotion, and (3) the utilization of emotions in solving problems, the Assessing Emotions Scale (AES) was developed (Schutte et al., 1998). Although the concept of EI was revisited with a four-branch model (Mayer, Caruso, \& Salovey, 2016), the AES has remained one of the most frequently used questionnaires investigating the different aspects of EI (see Supplementary material).

\section{El and ER Relationship}

The ability to successfully regulate emotions is a significant component of EI. Several studies have found a positive correlation between EI and cognitive reappraisal (CR) but a negative correlation between EI and suppression (Megías-Robles et al., 2019; Schutte, Manes, \& Malouff, 2009; Smieja, Mrozowicz, \& Kobylinska, 2011). Similarly, a higher level of EI was associated with a more frequent use of reappraisal and less frequent use of suppression (Cabello, Salguero, Fernández-Berrocal, \& Gross, 2013).

Peña-Sarrionandia, Mikolajczak, and Gross (2015) integrated the concept of EI into a process-model. They suggest that different ER patterns should be categorized into distinct stages of EI. High EI individuals regulate at the earliest point of the emotional process; thus, they show more antecedentfocused regulation (Schutte et al., 2009). They experience a confrontation with negative emotions and do not typically avoid affective states. In an embarrassing situation, they do their best to alter the circumstances and change the emotional consequences of the situation (Schutte et al., 2009). Trait EI is associated with increased perception of quality and quantity of social support (Mikolajczak, Luminet, Leroy, \& Roy, 2007), and high EI individuals take advantage of it. If the direct attempt to alter the situation is unsuccessful, they try to induce changes in an indirect way (e.g., they express their actual feelings). If high EI individuals have no way of altering the situation, they sustain their belief to handle the situation instead of rumination (Petrides, Pérez-Gonzaález, \& Furnham, 2007). They try to perceive the situation differently in order to alter their own feelings. As the ER process begins at a very early stage, there is no need to suppress emotional behavior or to neutralize emotions in a maladaptive way (e.g., by alcohol, drugs, intensified eating, or aggression; Peña-Sarrionandia et al., 2015).

Due to the fact that different types of ER processes result in various outcomes, the consequences of a certain event, for instance triggering an emotion, can induce distinctive effects in people with a high or low level of EI. People with high EI regulate their emotions more effectively than those with lower EI. They operate ER strategies in a flexible and adaptive way and take long-term advantages into consideration, both from their own perspective and in terms of social relationships (Peña-Sarrionandia et al., 2015). In sum, EI in the context of ER reveals that people with high EI are open to emotions, are able to effectively interpret them, and can apply this knowledge effectively when needed.

Using the experience sample method, Bucich and MacCann (2019) found that self-rated EI predicts more social sharing, direct situation modification, and reappraisal, while ability EI does not predict any of the five ER strategies.

Besides the process model of ER, special scientific interest has been dedicated to maladaptive or dysfunctional ER because of its clinical relevance. From a clinical perspective, maladaptive ER refers to a persistent pattern of deficits, which may affect the full range of emotional processing, including experiencing and expressing emotions (Sheppes, Suri, \& Gross, 2015). Gratz and Roemer (2004) developed the Difficulties in Emotion Regulation Scale (DERS), a multidimensional self-report measure of emotion dysregulation. This integrative conceptualization of ER involves not only the modulation of emotional arousal, but also the awareness, understanding, and acceptance of emotions, and the ability to control impulsive behavior and to act flexibly toward the desired goals (see Supplementary material). While EI has been integrated into the process model of ER, there is a gap in the literature on how the different EI components relate to ER difficulties resulting in either an adaptive or maladaptive functioning. One of our aims in this study is to explore the associations between different components of EI and the fields of ER difficulties.

Regardless of EI and ER, gender has a significant influence on emotional processes from perception, through appraisal, to regulation. Conflicting results, however, have been disclosed with regard to gender differences. Most researches have shown that women score higher on EI self-reports (e.g., Cabello, Sorrel, Fernández-Pinto, Extremera, \& FernándezBerrocal, 2016) and use both a larger and a wider variety of ER strategies than men (Tamres, Janicki, \& Helgeson, 2002). Moreover, women have higher means on EI dimensions that detect the use, perception, and expression of emotions such as Emotionality (Trait EI Questionnaire; Mikolajczak et al., 2007), and Use of emotion (WLEIS; Whitman, Van Rooy, Viswesvaran, \& Kraus, 2009). However, other studies have either demonstrated no gender differences (Pardeller, FrajoApor, Kemmler, \& Hofer, 2017), or advantages of men (Kong, 2017; Mikolajczak et al., 2007). Exploring the interaction between gender, EI and habitual use of ER, Smieja et al. (2011) found that emotionally intelligent males used reappraisal more frequently compared to women with high EI. In contrast, analyzing the suppression strategy, the gender 
difference appeared in the case of low EI. Men with a lower level of EI applied suppression more regularly than women with a similar extent of EI.

\section{The Current Study}

Successful ER, including CR, is described as a flexible and adaptive process of regulation that is logically consistent with internal goals (Mayer \& Salovey, 1995; Peña-Sarrionandia et al., 2015). One limitation of the above-mentioned studies is that in most cases only self-report measures have been used. CR performance should be verified with other methods besides surveys. In former ER studies that had used methods other than self-report measures, the primary goal was to compare different ER strategies (e.g., suppression vs. reappraisal). Participants were instructed to increase/decrease their affects or they could use any reappraisal strategies in order to decrease the unpleasantness. Pictures were presented twice with different instructions: for example, "view/watch," "suppress," and "reappraise" (for a meta-analysis of experimental conditions and stimuli, see Frank et al., 2014).

To our knowledge, no experimental setting exists where (1) flexibility is used for shifting from one affective state to another while using a cognitive ER strategy; and (2) participants use a goal-directed strategy to guide the ER process. Flexibility should be operationalized by a variable where participants can rate the subjective difficulty of changing their affective state. Goal-directed instructions can be provided by creating specific contexts using pictures with descriptive captions.

In our study, we apply self-report measures and an experiment at the same time: We use questionnaire data in order to describe participants' emotional characteristics (i.e., EI and ER difficulties), while the experiment has been designed to follow an ER process (i.e., a CR process where a stimulus interpretation had to be reframed by another). We operationalize $\mathrm{CR}$ in a guided picture viewing task (CR task), where two captions for the same picture offer different interpretations of a scene, consequently inducing two different emotional atmospheres. Emotional reactions are collected on two affective dimensions: valence and arousal. Valence ratings reveal whether the perceiver finds the picture pleasant or unpleasant. Arousal ratings indicate the level of emotional intensity that the perceiver feels (low or high level of arousal). Affective ratings are collected twice (before and after reappraisal) and are compared in order to see the changes. We used this complex research design of questionnaires and an experiment to gain a better understanding of how CR task performance in the experiment (i.e., the comparison of the affective ratings before and after reappraisal) is modified by individual emotional characteristics (i.e., components of EI and difficulties in different fields of ER).
Going beyond the novel methodological issue in the current study (i.e., the emotional atmosphere is created by the use of picture captions that induce different emotions within the different contexts), it is much less known how individual characteristics such as EI and different capacities in ER influence the affective changes while one uses a cognitive ER strategy. Does EI (and its components) have a different impact on affective responses compared with the effect of ER? In what direction do they moderate the affective change during CR? Does this moderating effect increase or decrease the affective ratings? Which affective dimension (valence, arousal, or both) is influenced by them? In other words, the question is still how the subfields of ER and the components of EI moderate the affective changes during a $\mathrm{CR}$ process in which an emotional event induces an initial affective response, and how this emotional response is subsequently modified via $\mathrm{CR}$.

\section{Aims, Research Questions, and Hypotheses}

In this study, we empirically reveal the EI and ER relationship using a CR task and self-report measures. One of the aims is to explore the ER-EI relationship, per se, and their specific associations with task-related variables (e.g., affective ratings, subjective level of task difficulty). Thus, we hypothesize (H1) that EI contributes to less difficulties in ER; that is, we expect a strong association between ER difficulties and EI. Moreover, given that both EI and difficulties in regulation rely on the perception and evaluation of emotional cues, we expect that ER and EI as individual characteristics impact CR task performance. We hypothesize task-specific correlations with $\mathrm{CR}$ task performance (H2). This hypothesis is divided into three parts that describe the relationship of task-related variables and EI (H2a); the relationship of task-related variables with ER difficulties (H2b); and the relationship of task-difficulty and ER and EI (H2c). More specifically, as high EI individuals are open to emotions including unpleasant ones, we expect lower valence ratings (i.e., more unpleasant) and high arousal (H2a). Furthermore, because ER also plays a crucial role in the appraisal of emotional stimuli, we expect that people with low scores on the ER difficulty scale (DERS) are more reactive in valence and arousal; that is, we hypothesize that ER difficulties correlate with a high level of valence and a low level of arousal (H2b). Finally, people with high EI and fewer ER difficulties are more skilled in regulating their taskspecific affective state (i.e., they have fewer difficulties in the $\mathrm{CR}$ task, so they give lower ratings on the $\mathrm{CR}$ task difficulty scale; $\mathrm{H} 2 \mathrm{c}$ ).

As this study primarily represents experimental research, we examine the changes in the affective ratings before and after CR while controlling for gender. We expect women to be more sensitive to emotional stimuli than men. We hypothesize (H3) that - compared with men - women report more unpleasant and more intense emotions to the affective stimuli. 
Furthermore, we investigate the moderating role of individual ER and EI characteristics on these affective changes. Our research question is how individual differences in ER and EI moderate changes in affective response.

\section{Materials and Methods}

\section{Participants}

Our sample consisted of 120 participants (60 men). Most of them were full-time university students from different faculties (medical studies, law, natural sciences, information technology [IT] and engineering, social sciences, and humanities) living in several geographical (mainly in the western and central) regions of Hungary. Their average age was 23.01 ( $S D=$ 5.03) years; the youngest participant was 18 , and the oldest was 45 . Participation was voluntary; the participants were not paid.

\section{Measures}

\section{El (AES-Hu)}

We used the Hungarian version of the AES (AES-HU; Kun, Balazs, Kapitany, Urban, \& Demetrovics, 2010) of the 33item questionnaire of Schutte et al. (1998). Participants indicated their answers on a 5-point scale, where 1 represented disagreement ("It is not at all typical of me.") and 5 represented agreement ("It is really typical of me."). The Hungarian study (Kun et al., 2010) confirmed the three-factor structure: (1) appraisal of emotions (AE); (2) optimism and regulation of emotions (ORE); and (3) intrapersonal and interpersonal utilization of emotions (UE). The internal consistency of the Hungarian version proved to be adequate (Cronbach's $\alpha$ is .882 for the total scale; .732 for AE; .712 for ORE; and .749 for UE).

\section{Difficulties in ER (DERS)}

The Hungarian version of Gratz and Roemer's (2004) Difficulties in Emotion Regulation Scale was adapted and validated by Kökönyei, Urbán, Reinhardt, Józan, and Demetrovics (2014). It consists of 36 items and six subscales: (1) non-acceptance of emotional responses (NonAcceptance), (2) difficulties engaging in goal-directed behavior (Goals), (3) impulse control difficulties (Impulse), (4) lack of emotional awareness (Awareness), (5) limited access to emotional regulation strategies (Strategies), and (6) lack of emotional clarity (Clarity). The reliability factors of the subscales were sufficient (Cronbach's alphas range from .73 to .86). Participants gave their answers on a 5-point Likert scale
( $1=$ almost never; $5=$ almost always $)$. High scores indicated greater emotional dysregulation.

\section{Emotional Stimuli}

We selected emotional stimuli from the International Affective Picture System (IAPS) database (Deak, Csenki, \& Gy, 2010; Lang, Bradley, \& Cuthbert, 2005). The colorful pictures induced different emotions. Participants evaluated their emotional states on two affective dimensions: valence (unpleasant-pleasant) and arousal (relaxed-aroused). Their responses were evaluated according to a 9-point scale on a visual analog scale (Self-Assessment Manikin). Low scores on the valence dimension indicate negative and unpleasant feelings, while high values represent positive and pleasant emotions. Low scores on the arousal dimension represent a calmed, relaxed, or bored state, while high scores imply an intensified alertness, high activity level, and piqued attention. After a series of pilot studies, we selected 15 pictures depicting social-emotional situations. The following images were used in the CR task: 2480, 2616, 2691, 4598, 5629, $6212,6250,6360,6530,6825,6836,8010,9050,9102$, and 9421.

\section{CR Task and Procedure}

We utilized a CR task (Deak et al., 2017) where pictures were presented one by one with two different captions displayed one after the other. Participants had to look at the picture captions and let their emotions flow. We drew their attention to the fact that the same picture was going to be displayed with a different caption and the new caption would allow them to reappraise the situation from another point of view. For example, the picture of an old man standing by the window was titled "Goodbye"; however, in the second presentation it was titled "Waiting." By attaching the same visual stimulus to two distinctive contexts, participants had to perform CR.

During the CR task, participants evaluated the images labeled with titles regarding valence and arousal; they also ranked how difficult the reappraisal had been on a 9-point scale. If the participants gave a high rating, it meant that it was difficult for them to interpret the picture from the second point of view, and so the switch between the two meanings was complicated.

After informed consent was obtained from all individual participants, they performed the CR task first and then completed the questionnaires. All procedures were in accordance with the ethical standards of the United Ethical Review Committee for Research in Psychology (EPKEB; reference number 2017/126) and with the 1964 Declaration of Helsinki. 


\section{Data Analysis}

Power analysis was conducted using G-Power 3.1.9.4 to determine whether the sample size was adequate $(1-\beta=.95$; $\alpha=.05 ; \mathrm{f} 2=.15)$. Power analysis revealed that the current sample size $(N=120)$ exceeded the minimum required number of participants (66).

Statistical analysis was conducted using SPSS 19. A normal distribution was confirmed by the Kolmogorov-Smirnoff test. With regard to the general and task-specific associations of EI and ER, Pearson's correlation was computed to assess interscale correlations between the questionnaire data (AES total and subscale scores; DERS total and subscale scores) and task-related variables (valence and arousal ratings before and after reappraisal, task difficulty). A series of analyses of covariance (ANCOVAs) was used to assess the impact of individual characteristics (i.e., gender, fields of ER difficulties, and components of EI) on the affective changes between the emotional responses before and after reappraisal in the $\mathrm{CR}$ task. Valence and arousal were the within-subjects factors, respectively, with two levels (before and after reappraisal); gender was the between-subjects factor (two levels: men and women). Individual characteristics (scores on DERS and AES subscales) were continuous variables and added as covariates.

The datasets generated and analyzed during the current study are available from the corresponding author upon request.

\section{Results}

First, we introduce the interscale correlations of ER and EI, then their task-specific associations with the five task-related variables (valence before/after, arousal before/after, and task difficulty). Second, the ANCOVA results are presented with ER and EI as individual moderators on the affective changes. Here we specify (1) which subscale has a significant role (2) on which emotional dimension (valence, arousal or both), and (3) in what direction it effects the affective change (increase or decrease). Third, since gender has a significant overall impact, we summarize the gender-related results in a separate section.

\section{General and Task-Specific Associations of ER and EI}

There was a significant negative association between the DERS total and the AES total scores (Table 1). This means that participants who reported higher EI scores (i.e., have better skills in emotion recognition and deeper knowledge about the generation and course of emotion) were likely to have fewer ER difficulties (i.e., using adaptive ER strategies successfully).

There were mild to moderate negative correlations between the AES total scores and the following DERS subscales:
Impulse, Awareness, Strategies, and Clarity. Hence, attention to the emotional signals, having access to ER strategies, taking control over emotions, and the presence of emotional clarity all contributed to a higher level of EI.

The AES Appraisal scores negatively correlated with DERS Awareness and DERS Clarity. These results indicate that better skills in identification and interpretation of emotions were in line with fewer ER difficulties in having access to one's own affective states and having a clear understanding of them. The AES Optimism and ER subscale scores negatively correlated with the DERS total score, as well as with all subscales. These results validate the two self-report questionnaires showing that they measured similar constructs. On the other hand, our results indicate that the more positive mood an individual had due to successful ER processes, the less difficulties s/he had experienced. Moreover, AES Utilization scores negatively correlated with DERS Awareness and DERS Clarity. This means that fewer difficulties in understanding emotions contribute to the utilization of emotions in intrapersonal and interpersonal situations.

In order to investigate the task-specific associations between the personality variables (ER difficulties, EI) and CR task performance, we computed another series of correlations (Table 1). Valence ratings before reappraisal correlated negatively with the AES total and Appraisal and Utilization, and positively with the DERS Awareness. Valence ratings after reappraisal showed a tendency for a negative correlation with the AES total score, and a significant positive correlation with the DERS Impulse. The Arousal ratings before reappraisal correlated positively with the DERS Non-Acceptance and negatively with the DERS Awareness, while the Arousal ratings after reappraisal correlated negatively only with the DERS Awareness. Task difficulty correlated with the two subscales measuring ER, respectively: positively with the DERS Strategies subscale and negatively with the AES Optimism and ER subscale at tendency level $(p=.055)$.

These results demonstrate that participants with higher levels of EI, in addition to a lower level of ER difficulties (i.e., fewer difficulties in paying attention to emotions), gave lower (i.e., more unpleasant) ratings on the valence dimension when they saw the pictures for the first time. However, valence ratings after reappraisal were higher (i.e., more pleasant) when the person has more ER difficulties in taking control over his/her emotions. The positive correlation between the arousal ratings before reappraisal and the DERS Non-Acceptance means that participants with higher scores on this subscale had higher arousal ratings, thus experiencing more intense emotions. From the results showing negative association between the Arousal before reappraisal, Arousal after reappraisal, and the DERS Awareness, we can see that participants who had more ER difficulties in paying attention to emotions reported less intense arousal. 


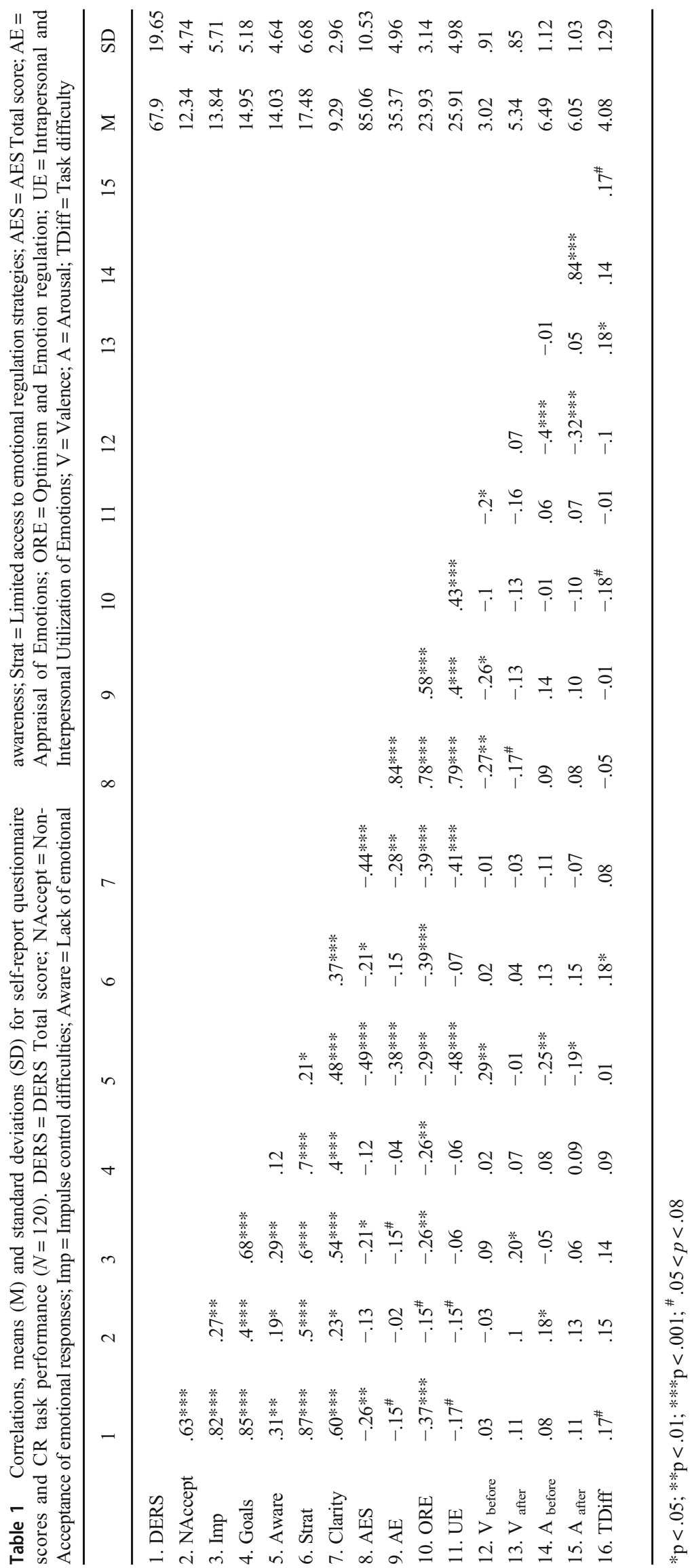




\section{The Moderating Effect of ER and El as Individual Characteristics on Affective Changes in the CR Task}

DERS Impulse and DERS Clarity as covariates had a highly significant effect on the affective change on valence dimension (Table 2). An unclear understanding of emotions (Clarity) decreased valence ratings both before and after reappraisal; that is, individuals with problems differentiating emotions and knowing what they feel had more unpleasant ratings at both phases of the CR task. We conclude that difficulties in clarity resulted in a bias toward unpleasant feelings regardless of CR. Hence, its moderatory role was general on valence dimension. In contrast, a low level of control over emotions (Impulse) increased the valence ratings after reappraisal. Specifically, the pictures became less unpleasant for impulsive individuals after regulation occurs (Table 3 ).

Regarding the changes in arousal ratings, the Arousal $\times$ DERS Impulse interaction was statistically significant $\left(F(1,109)=5.75, p<.05 ; \eta^{2}=.05\right)$. Thus, the effect of gender on arousal ratings before and after reappraisal depended on the DERS Impulse score. The results of a further analysis for women showed a weak negative correlation $(r=-.33$, $p<.05$ ) between DERS Impulse, Arousal before, and Arousal after. In other words, women with lower DERS Impulse scores (i.e., with fewer problems controlling emotions) had higher arousal ratings (i.e., more intense emotions) at the initial phase of the CR task. This result was only specific for women; there was no correlation in men.
As a covariate, DERS Awareness had a marginally significant impact on changes in arousal ratings $(p=.07)$. It decreased arousal ratings before reappraisal: Inattention to emotions (i.e., higher scores on DERS Awareness) resulted in less intensive affective ratings.

The AES components as covariates had only a weak impact on affective changes compared with DERS subscales as covariates. AES Utilization had a significant impact on valence changes. Specifically, it decreased valence ratings after reappraisal: They became more unpleasant if one had a stronger ability to utilize emotions both in the intrapsychic dimension and in interpersonal situations.

\section{Affective Changes Moderated by Gender}

Regardless of the effect of individual characteristics, there was a significant difference between the valence ratings before and after reappraisal $(F(1,109)=13.29, p<.001$; $\left.\eta^{2}=.11\right)$. Pictures with the first caption were unpleasant $(M=3.02 ; S D=0.91)$ and became less unpleasant (i.e., neutralized) with the second caption $(M=5.34 ; S D=$ $0.85)$. Women rated them as more unpleasant than men $\left(F(1,109)=7.54, p<.01 ; \eta^{2}=.07\right)$, and as it turned out from the significant Gender $\times$ Valence interaction $\left(F(1,109)=41.44, p<.001 ; \eta^{2}=.28\right)$, women rated the first pictures more unpleasant $(M=2.55 ; S E=0.10)$ than men $(M=3.50 ; S E=0.10)$, while the second ratings were

Table 2 Analyses of covariance (ANCOVAs) for affective changes in valence and arousal, respectively, in the cognitive reappraisal (CR) task using AES and DERS subscales as covariates

\begin{tabular}{|c|c|c|c|c|c|c|c|c|c|c|}
\hline & \multicolumn{5}{|c|}{ Valence } & \multicolumn{5}{|c|}{ Arousal } \\
\hline & $\mathrm{D} f$ & Ms & $\mathrm{F}$ & $\mathrm{p}$ & $\eta^{2}$ & $\mathrm{D} f$ & Ms & $\mathrm{F}$ & $\mathrm{p}$ & $\eta^{2}$ \\
\hline Main effect of Affective change (before and after CR) & 1 & 6.77 & 13.29 & $<.001 * * *$ & .11 & 1 & .23 & 1.25 & .27 & .01 \\
\hline Gender & 1 & 4.91 & 7.53 & $<.01 * *$ & .07 & 1 & 17.69 & 9.43 & $<.01 * *$ & .08 \\
\hline Gender $\mathrm{x}$ Affective change interaction & 1 & .51 & 41.44 & $<.001 * * *$ & .28 & 1 & .07 & .37 & .54 & $<.01$ \\
\hline \multicolumn{11}{|l|}{ DERS } \\
\hline Non-Acceptance & 1 & .27 & .42 & .52 & $<.01$ & 1 & 1.81 & .96 & .33 & .01 \\
\hline Impulse & 1 & 6.90 & 10.61 & $<.01 * *$ & .09 & 1 & .17 & .09 & .77 & $<.01$ \\
\hline Goals & 1 & .01 & $<.01$ & .94 & $<.01$ & 1 & .03 & .02 & .90 & $<.01$ \\
\hline Awareness & 1 & .13 & .20 & .66 & $<.01$ & 1 & 5.90 & 3.15 & $.07^{\#}$ & .03 \\
\hline Strategies & 1 & .80 & 1.22 & .27 & .01 & 1 & 3.14 & 1.68 & .20 & .02 \\
\hline Clarity & 1 & 6.30 & 9.69 & $<.001 * * *$ & .08 & 1 & 2.53 & 1.35 & .25 & .01 \\
\hline \multicolumn{11}{|l|}{$A E S$} \\
\hline Appraisal & 1 & 1.26 & 1.94 & .17 & .02 & 1 & .11 & & .81 & $<.01$ \\
\hline Regulation & 1 & .04 & .05 & .82 & $<.01$ & 1 & .06 & & .86 & $<.01$ \\
\hline Utilization & 1 & 2.78 & 4.28 & $.04 *$ & .04 & 1 & 2.05 & & .30 & .01 \\
\hline Error & 109 & & & & & 109 & & & & \\
\hline
\end{tabular}

$* \mathrm{p}<.05 ; * * \mathrm{p}<.01 ; * * * \mathrm{p}<.001 ;{ }^{\#} .05<\mathrm{p}<.08$ 
Table 3 Estimated parameters and effect sizes of gender and subscales of AES and DERS as moderators of affective changes in the CR task based on the ANCOVAs

\begin{tabular}{|c|c|c|c|c|c|c|c|c|c|c|c|c|c|c|c|}
\hline & \multicolumn{3}{|c|}{$\begin{array}{l}\text { Valence ratings before } \\
\text { cognitive reappraisal }\end{array}$} & \multicolumn{3}{|c|}{$\begin{array}{l}\text { Valence ratings after } \\
\text { cognitive reappraisal }\end{array}$} & \multicolumn{3}{|c|}{$\begin{array}{l}\text { Arousal ratings before } \\
\text { cognitive reappraisal }\end{array}$} & \multicolumn{3}{|c|}{$\begin{array}{l}\text { Arousal ratings after } \\
\text { cognitive reappraisal }\end{array}$} & \multicolumn{3}{|c|}{ CR Task difficulty } \\
\hline & $\beta$ & $\mathrm{t}$ & $\eta^{2}$ & $\beta$ & $\mathrm{t}$ & $\eta^{2}$ & $\beta$ & $\mathrm{t}$ & $\eta^{2}$ & $\beta$ & $\mathrm{t}$ & $\eta^{2}$ & $\beta$ & $\mathrm{t}$ & $\eta^{2}$ \\
\hline Gender & .96 & $6.67 * * *$ & .29 & -.33 & $-2.11^{*}$ & & -.63 & $-3.05^{* *}$ & .08 & -.55 & $-2.81 * *$ & .07 & -.57 & $-2.27^{*}$ & .05 \\
\hline \multicolumn{16}{|l|}{$A E S$} \\
\hline Appraisal & -.02 & -1.02 & .01 & -.02 & .18 & .01 & .01 & .39 & $<.01$ & $<.01$ & .07 & $<.01$ & .02 & .68 & $<.01$ \\
\hline Regulation & .01 & .30 & $<.01$ & -.02 & .42 & $<.01$ & -.01 & -.26 & $<.01$ & $<-.01$ & -.08 & $<.01$ & -.10 & $-1.83^{\#}$ & .03 \\
\hline Utilization & -.02 & -.99 & .01 & -.04 & $-2.96 * *$ & .04 & -.03 & -1.21 & .01 & -.02 & -.78 & $<.01$ & .01 & .47 & $<.01$ \\
\hline \multicolumn{16}{|l|}{ DERS } \\
\hline Non-Acceptance & $<-.01$ & -.17 & $<.01$ & .02 & 1.08 & .01 & .03 & 1.20 & .01 & .02 & .66 & $<.01$ & .03 & .92 & .01 \\
\hline Impulse & .03 & 1.72 & .03 & .06 & $3.10 * *$ & .08 & -.03 & -.98 & $<.01$ & .01 & .44 & $<.01$ & -.04 & .95 & .01 \\
\hline Goals & .01 & .50 & $<.01$ & -.01 & -.34 & $<.01$ & .01 & .17 & $<.01$ & -.01 & -.42 & $<.01$ & .01 & -1.14 & .01 \\
\hline Awareness & .03 & 1.50 & .02 & -.02 & -.72 & .01 & -.05 & $-1.91^{\#}$ & .03 & -.04 & -1.47 & .02 & $<.01$ & .12 & $<.01$ \\
\hline Strategies & -.01 & -.33 & $<.01$ & -.02 & -1.28 & .02 & .03 & 1.29 & .02 & .03 & 1.18 & .01 & .02 & .55 & $<.01$ \\
\hline Clarity & -.06 & $-1.91^{\#}$ & .03 & -.1 & $-2.71 * *$ & .06 & -.05 & -1.06 & .01 & -.05 & -1.16 & .01 & -.02 & -.39 & $<.01$ \\
\hline
\end{tabular}

$* \mathrm{p}<.05 ; * * \mathrm{p}<.01 ; * * * \mathrm{p}<.001 ;{ }^{\#} .05<\mathrm{p}<.08$

quite similar in both groups (women: $M=5.51 ; S E=0.11$; men: $M=5.18 ; S E=0.11$; Table 2). For the adjusted and unadjusted means and variability for valence see Table 4 .

Regarding the affective change between the arousal ratings before and after reappraisal, the decrease from $6.49(S D=1.12)$ to $6.05(S D=1.03)$ was not significant $\left(F(1,109)=1.25, p>.05 ; \eta^{2}=.01\right)$. However, there was a gender difference regardless of the CR task phases. Women experienced more intense emotions before $(M=6.80, S E=0.14)$ and after $(M=6.32, S E=0.13)$ reappraisal than men (before: $M=6.18 ; S E=0.14$; after: $\left.M=5.77 ; S E=0.13 ; F(1,109)=9.43 p<.01 ; \eta^{2}=.08\right)$. The Gender $\times$ Arousal interaction was not significant (Table 2). For the adjusted and unadjusted means and variability for arousal see Table 4 .

Table 4 Adjusted and unadjusted gender means and variability for affective changes in the CR task using AES and DERS subscales as covariates

\begin{tabular}{|c|c|c|c|c|c|c|c|c|c|}
\hline & & \multicolumn{4}{|c|}{ Unadjusted } & \multicolumn{4}{|c|}{ Adjusted } \\
\hline & & \multicolumn{2}{|c|}{ Before } & \multicolumn{2}{|c|}{ After } & \multicolumn{2}{|c|}{ Before } & \multicolumn{2}{|l|}{ After } \\
\hline & & M & $\mathrm{SD}$ & $\mathrm{M}$ & $\mathrm{SD}$ & M & SE & M & $\mathrm{SE}$ \\
\hline \multirow[t]{2}{*}{ Valence } & Men & 3,55 &, 77 & 5,21 &, 71 & 3,50 &, 10 & 5,18 &, 11 \\
\hline & Women & 2,5 &, 70 & 5,48 & ,95 & 2,55 &, 10 & 5,51 &, 11 \\
\hline \multirow[t]{2}{*}{ Arousal } & Men & 6,13 & 1,12 & 5,73 & 1,00 & 6,18 &, 14 & 5,77 &, 13 \\
\hline & Women & 6,85 & 1,00 & 6,36 & 1,03 & 6,80 & ,14 & 6,32 & ,13 \\
\hline
\end{tabular}

\section{Discussion}

\section{El and ER Relationship}

The purpose of this study was to rethink the concept of EI in the framework of ER and to identify individual differences that contribute to a successful ER process when an individual changes his/her affective state with a CR strategy. One of our aims was not only to describe the interaction of EI and ER through self-report measures but to investigate them as moderators of affective changes in an empirical research context.

Compared with former studies that have shown EI is associated with more adaptive forms of ER such as reappraisal (e.g., Cabello et al., 2013; Megías-Robles et al., 2019; Schutte et al., 2009; Smieja et al., 2011), we demonstrated links between the components of EI and different ER difficulties. As expected (H1), we found that strong skills in emotion recognition and deep knowledge about the generation and course of emotion contribute to fewer ER difficulties. More precisely, the higher the level of EI an individual shows, the less difficulty s/he has in attending to emotional signs, understanding and controlling them, recognizing whether the affective state is appropriate and/or must be regulated, and applying successful ER strategies.

Specifically, as it appeared from the significant correlations, ER difficulties in awareness and clarity are associated with two components of EI, namely appraisal and utilization. Indeed, better skills in recognizing the importance and 
relevance of emotional signs, more attention to them, and advanced identification of the experienced emotion contribute to adequate evaluation and utilization of emotional processes. These findings are consistent with previous studies (Boden \& Thompson, 2015; Joseph \& Newman, 2010) and suggest that the essential parts of successful regulation are the adequate perception, attention, and understanding of emotional cues. The cascading model of EI developed by Joseph and Newman (2010) differentiates three main elements called emotion perception, emotion understanding, and ER. This conceptual framework states that these subdimensions are sequentially related to each other and show a causal chain. Therefore, the prerequisite of regulation is perception and understanding of affects. It means that without appropriate attention to and identification and understanding of emotions, there is no effective ER.

The association between regulation and awareness that we found has also been confirmed by Boden and Thompson (2015) in their structural model of emotional awareness. The authors examined the connection between the four facets of awareness - type clarity, source clarity, involuntary attention, and voluntary attentionand reappraisal. Relying on their results, Boden and Thompson (2015) stated that identifying emotions, understanding them, and knowing the reason why they emerged facilitate the adequate use of emotional reappraisal. Accordingly, these findings highlight the required role of attention to and identification of emotional cues in the process of regulation, which overlaps with our results concerning the connection between awareness, emotional clarity, and AES components (Appraisal and Utilization). Hence, advanced appraisal skillsincluding the ability to utilize the previous experience of emotional situations - are conducive to stronger affective control functions and beneficial behavior. With our negative correlations between all fields of ER difficulties and the positive attitude toward a successful ER process (i.e., ORE scale), we confirm Shahzad, Begum, and Khan's findings (Shahzad, Begum, \& Khan, 2013) that higher trait EI is associated with fewer behavioral problems. Consequently, executive functions are being applied when emotions are used in problem-solving (intrapersonal utilization) or when socio-emotional skills support everyday interactions (interpersonal utilization; e.g., maintaining long-term goals, inhibiting non-goal-directed actions, neglecting irrelevant information).

Moreover, a positive attitude toward the success of ER and positive emotions is connected to a better identification and differentiation of emotions, as well as to a more successful outcome of ER. Based on our results, we agree with previous findings (Schutte et al., 2009) that high EI individuals are active and they change the situation either to arouse emotions or to avoid them.

\section{Components of El and Difficulties of ER as Moderators}

We demonstrated the general and task-specific associations of ER difficulties and EI. Given that we investigated the ER-EI interaction through self-report measures as well as their impact in an experimental setting (i.e., on affective ratings in a CR task), in this section we explain the moderating effect of individual differences in EI and ER on affective changes during CR.

The negative correlations between the initial valence ratings (i.e., valence before reappraisal) and EI partly confirmed our hypothesis $(\mathrm{H} 2 \mathrm{a})$ that a high level of EI contributes to lower valence ratings (i.e., more unpleasant feelings). The ANCOVA results revealed that AES Utilization had a significant decreasing impact on valence changes in the CR task (i.e., valence ratings after reappraisal became more unpleasant if one has stronger ability to utilize emotions). As an EI component, the intra- and interpersonal utilization of emotions enables one to use emotions in problem solving. Although utilization is based on the adaptive usage of arousal (Izard et al., 2011), there is still a question of why it maintains emotions as unpleasant after the reappraisal.

Contrary to our expectations (H2a), we did not find a connection between EI and arousal. The ANCOVA results also confirmed the lack of EI impact on arousal changes. A high level of arousal related only to ER difficulties at tendency level, but not at all to EI. Therefore, based on the results we suggest that EI may have an impact on valence as a construct of emotional perception and interpretation of emotional signals, while individual differences in ER (i.e., difficulties in different fields of ER) have an effect on both valence and arousal. Consequently, ER seems to have a broader effect than EI.

As we can see, ER difficulties impact changes in valence and arousal ratings both before and after reappraisal. The initial appraisal of an emotional stimulus (i.e., identifying them as pleasant or unpleasant, arousing or not) is moderated by difficulties in paying attention to emotions (Awareness), and in identifying them (Clarity). When an individual uses a regulatory strategy such as CR, his/her emotions are influenced by individual factors like problems in understanding emotions (Clarity) and in controlling them (Impulse).

Clarity has a special function among the moderators. This is the only subscale that has an effect on affective responses both before and after ER in the same direction (i.e., unclear emotions decrease the valence ratings). Specifically, if one has problems in clearly identifying and understanding emotions, more unpleasant feelings are elicited regardless of the phase of ER. This moderating role of clarity on valence is supported by other findings (Nielsen \& Kaszniak, 2006; Vine \& Aldao, 2014). Unsuccessful ER caused by a lack of clarity may make individuals vulnerable to psychopathology. Vine and Aldao (2014) found that deficits in emotional clarity contribute to 
symptoms of depression, social anxiety, borderline personality, binge eating, and alcohol use. A question that remains is whether there is a causal relationship between the negativity bias due to difficulties in emotional clarity and one's vulnerability to emotional disorders. Further studies are required in this field.

The impact of ER difficulties in the field of Clarity, Impulse, and Awareness (see ANCOVA results) and the task-specific correlations with valence and arousal ratings have strengthened our $\mathrm{H} 2 \mathrm{~b}$ hypothesis. Our results are in line with former studies (Aluja et al., 2015) in which impulsivity correlated with positive valence and high arousal. We suggest that impulsivity serves ER by neutralizing the unpleasant emotion. An impulsive person may find it difficult to control intense affective states. S/he may be motivated to get rid of internal tension as soon as possible. As any decrease in arousal level might be rewarding for him/her, the picture with the second caption (i.e., the same stimulus in a new context) evokes less unpleasant emotions. We think that the new context overwrites and diminishes the initial affective response. We argue that if the negative emotional atmosphere (induced by the first caption) is barely acceptable for the person, this stress might be added to the intensity of the first picture resulting in even higher subjective ratings of arousal.

Additionally, we found a significant Arousal $\times$ Impulse interaction controlled for gender: Impulse moderates the change in arousal ratings when gender is also considered. We claim that fewer problems in controlling emotions leads to more intense emotion before reappraisal for women. If impulsive persons suppress their affective expressions, they probably can subjectively feel more intense feelings. Women who have less control over their emotions and, for example, give verbal comments when they are touched by the picture (e.g., "Oh, my God!"; "It is so sad!") can relieve their tension and do not feel the residual so intense.

While impulsivity problems decrease unpleasantness (i.e., neutralizing), difficulties in attending to affective signals and being aware of emotions (Awareness) decreases the intensity of emotions. Specifically, if the person is unaware of a current affective state, it would be out of focus; therefore, the arousal level would not increase. The positive correlation between attention to emotions and affect intensity has been demonstrated in previous studies (Gohm \& Clore, 2002; Thompson, Dizén, \& Berenbaum, 2009). In other words, an individual who pays more attention to his/her emotions experiences higher level of intensity.

Our findings on negative correlations between arousal and ER difficulties (H2b; i.e., with more intense emotions there are fewer ER difficulties) overlap with the results of Barrett, Gross, Christensen, and Benvenuto (2001), who found that those individuals who experienced their emotion at greater intensity distinguished negative emotions more precisely and used more ER. The authors concluded that the ability to differentiate negative emotions contributes to the wider range of ER strategies. This phenomenon is in accordance with our finding: If the person is unable to identify emotions and differentiate them (Clarity) and the effectiveness of ER strategies is also not perceived, then the initial unpleasant feelings remain even after the reappraisal.

Our examination of how the individual factors are linked to the CR task difficulty (i.e., how difficult it was to switch from one emotional context to another one) revealed a congruent effect. The significant correlations supported the prediction $(\mathrm{H} 2 \mathrm{c})$ that EI and ER correlate with the performance of the CR task: The ability to have better access to strategies (ER component) and the positive attitude toward the regulation of emotions (EI component) contributed to the feeling of less difficulty in reappraising. However, their contribution is weak as a moderator (i.e., $p_{\mathrm{AES} O \mathrm{ORE}}=.07$ ), and further confirmatory studies are urged.

Overall, our study described the moderating role of EI and ER in the subjective efficiency of reappraisal and their specific patterns during the process of regulation. We conclude that while EI contributes to the evaluation of the valence (pleasantness or unpleasantness), ER difficulties have an influence on the entire emotional process from the perception of emotional stimuli through the assessment of them until ER occurs. The distinctive role of EI and ER on affective ratings might come from their different construct and quality. Different EI components and ER strategies influence conscious changes in emotions in different ways.

\section{Gender Differences}

Gender differences must be considered when emotions are investigated. In line with previous research, we also found that women's emotions were more unpleasant and more intense than men's. In their study, Bradley, Codispoti, Sabatinelli, and Lang (2001) presented IAPS pictures and participants gave their ratings on valence and arousal dimensions. According to the results, women were more reactive to unpleasant materials: They rated these pictures more unpleasant and more arousing than the males did. A more recent study with a different set of affective pictures (Marchewka, Zurawski, Jednorog, \& Grabowska, 2014) also found that the correlation between valence and arousal was stronger for women than for men, especially for social stimuli (e.g., people, faces, and objects). These results might refer to women's bias toward unpleasant pictures; women tend to primarily rate unpleasant pictures more arousing than males. (For a meta-analysis, see Filkowski, Olsen, Duda, Wanger, \& Sabatinelli, 2017.)

As we hypothesized (H3), women's emotional reactions were more unpleasant and arousing compared with men's. The significant Valence $\times$ Gender interaction means that men's initial emotion was less unpleasant (i.e., closer to neutral) in the CR task compared with women's response. Their 
emotions were less negative; thus, the affective shift between before and after reappraisal is smaller. Although we did not find significant interaction in the case of Gender $\times$ Arousal, the significant main effect of gender shows women's more intense feelings than men's even after the regulation. Based on the changes on both affective dimensions (i.e., valence and arousal), we suggest that males' less unpleasant and less arousing affective state resulted in a smaller shift between before and after reappraisal. Consequently, the task of shifting from the first context to the second during the reappraisal was easier for men than for women.

\section{Conclusions and Limitations}

"While some theories define ER as an integral component of EI, others see EI as the general potential for ER" (Zysberg \& Raz, 2019, p. 202), our findings may provide a more sophisticated picture about the nature of the two research traditions. The current study is the first to demonstrate the moderating role of EI and ER difficulties on changes in affective responses (i.e., valence and arousal) during a CR task. Besides the frequently used self-report measures (e.g., Cabello et al., 2013; Megías-Robles et al., 2019; Schutte et al., 2009) and some experimental studies (Bucich \& MacCann, 2019; Zysberg \& Raz, 2019), our research provides a unique experimental design using a CR task. Compared with the examination of Zysberg and Raz (2019), who asked participants to self-induce emotional arousal then relax, we created a more direct, guided, and predetermined emotional context in which reappraisal takes place. In this controlled environment, we had the opportunity to observe the subjects' emotional states stepby-step (before and after ER) and follow-up the evolvement in the light of individual characteristics such as EI and ER.

Compared with previous works (e.g., Bucich \& MacCann, 2019; Cabello et al., 2013; Megías-Robles et al., 2019), the novel contribution of the present study is that we provide an explanation of how EI and ER difficulties use different routes (i.e., affective dimensions) and have their specific influence on the affective outcome: EI moderates changes in the affective dimension of valence through the intra-and interpersonal utilization of emotions, while individual differences in ER have a broader impact on both valence and arousal dimensions through emotional clarity, awareness, and impulse. We identified specific ways in which individual differences modify a successful ER process when using a CR strategy. Our work contributes to a better understanding of the relationship between the difficulties of ER and the components of EI; furthermore, we highlight their role as moderators in CR. Knowing what we feel and why we feel that supports a successful ER process through a proper identification of reactions to emotional stimuli.
As a potential limitation of our study, the different constructs in the same instrument (e.g., Awareness and Clarity of DERS) had different effects on the same dependent variable. Our findings are similar to former studies (e.g., Kökönyei et al., 2014) in which the Awareness subscale behaved differently compared with the other five DERS subscales. Further investigation is needed to clarify the conceptual differences and whether Awareness is more like an emotion perception process than an emotion regulatory one.

Supplementary Information The online version contains supplementary material available at https://doi.org/10.1007/s12144-020-01182-5.

Acknowledgments Open access funding provided by University of Pécs. This work was supported by the National Research, Development and Innovation Fund of Hungary under Grants K125437 and FK125007.

Author's Contribution All authors contributed to the study conception and literature review. Material preparation, data collection and analysis were performed by Barbara Bodrogi and Anita Deak. The first draft of the manuscript was written by Barbara Bodrogi and Anita Deak. All authors commented on previous versions of the manuscript, read and approved the final manuscript.

Funding This work was supported by the National Research, Development and Innovation Fund of Hungary under Grants K125437 (to Tamas Bereczkei) and FK125007 (to Anita Deak).

Data Availability On request from corresponding author.

\section{Compliance with Ethical Standards}

Conflict of Interest The authors declare that they have no conflict of interest.

Open Access This article is licensed under a Creative Commons Attribution 4.0 International License, which permits use, sharing, adaptation, distribution and reproduction in any medium or format, as long as you give appropriate credit to the original author(s) and the source, provide a link to the Creative Commons licence, and indicate if changes were made. The images or other third party material in this article are included in the article's Creative Commons licence, unless indicated otherwise in a credit line to the material. If material is not included in the article's Creative Commons licence and your intended use is not permitted by statutory regulation or exceeds the permitted use, you will need to obtain permission directly from the copyright holder. To view a copy of this licence, visit http://creativecommons.org/licenses/by/4.0/.

\section{References}

Aluja, A., Rossier, J., Blanch, Á., Blanco, E., Martí-Guiu, M., \& Balada, F. (2015). Personality effects and sex differences on the international affective picture system (IAPS): A Spanish and Swiss study. Personality and Individual Differences, 77, 143-148.

Antonakis, J., Ashkanasy, N. M., \& Dasborough, M. T. (2009). Does leadership need emotional intelligence? The Leadership Quarterly, 20(2), 247-261. 
Antonakis, J., \& Dietz, J. (2010). Emotional intelligence: On definitions, neuroscience, and marshmallows. Industrial and Organizational Psychology, 3(2), 165-170.

Barrett, L. F., Gross, J., Christensen, T. C., \& Benvenuto, M. (2001). Knowing what you're feeling and knowing what to do about it: Mapping the relation between emotion differentiation and emotion regulation. Cognition and Emotion, 15(6), 713-724.

Boden, M. T., \& Thompson, R. J. (2015). Facets of emotional awareness and associations with emotion regulation and depression. Emotion, 15(3), 399-410.

Bradley, M. M., Codispoti, M., Sabatinelli, D., \& Lang, P. J. (2001). Emotion and motivation II: Sex differences in picture processing. Emotion, 1(3), 300-319.

Bucich, M., \& MacCann, C. (2019). Emotional intelligence and day-today emotion regulation processes: Examining motives for social sharing. Personality and Individual Differences, 137, 22-26.

Cabello, R., Salguero, J. M., Fernández-Berrocal, P., \& Gross, J. J. (2013). A Spanish adaptation of the emotion regulation questionnaire. European Journal of Psychological Assessment, 29(4), 234 240

Cabello, R., Sorrel, M. A., Fernández-Pinto, I., Extremera, N., \& Fernández-Berrocal, P. (2016). Age and gender differences in ability emotional intelligence in adults: A cross-sectional study. Developmental Psychology, 52(9), 1486-1492.

Deak, A., Bodrogi, B., Biro, B., Perlaki, G., Orsi, G., \& Bereczkei, T. (2017). Machiavellian emotion regulation in a cognitive reappraisal task: An fMRI study. Cognitive, Affective, \& Behavioral Neuroscience, 17, 528-541.

Deak, A., Csenki, L., \& Gy, R. (2010). Hungarian ratings for the international affective picture system (IAPS): A cross-cultural comparison. Empirical Text and Culture Research, 4, 90-101.

Fan, H., Jackson, T., Yang, X., Tang, W., \& Zhang, J. (2010). The factor structure of the Mayer-Salovey-Caruso emotional intelligence test V2.0: A meta-analytic structural equation modeling approach. Personality and Individual Differences, 48, 781-785.

Filkowski, M. M., Olsen, R. M., Duda, B., Wanger, T. J., \& Sabatinelli, D. (2017). Sex differences in emotional perception: Meta-analysis of divergent activation. NeuroImage, 147, 925-933.

Frank, D. W., Dewitt, M., Hudgens-Haney, M., Schaeffer, D. J., Ball, B. H., Schwartz, N., Hussein, A. A., Smart, L. M., \& Sabatinelli, D. (2014). Emotion regulation: Quantitative meta-analysis of functional activation and deactivation. Neuroscience and Biobehavioral Reviews, 45, 202-211.

Gohm, C. L., \& Clore, G. L. (2002). Four latent traits of emotional experience and their involvement in well-being, coping, and attributional style. Cognition and Emotion, 2002, 16(4), 495-518.

Gratz, K. L., \& Roemer, L. (2004). Multidimensional assessment of emotion regulation and Dysregulation: Development, factor structure, and initial validation of the difficulties in emotion regulation scale. Journal of Psychopathology and Behavioral Assessment, 26, 41-54.

Gross, J. J. (1998). The emerging field of emotion regulation: An integrative review. Review of General Psychology, 2(5), 271-299.

Gross, J. J. (2015). Emotion regulation: Current status and future prospects. Psychological Inquiry, 26, 1-26.

Izard, C. E., Woodburn, E. M., Finlon, K. J., Krauthamer-Ewing, E. S., Grossman, S. R., \& Seidenfeld, A. (2011). Emotion knowledge, emotion utilization, and emotion regulation. Emotion Review, 3(1), 44-52.

Joseph, D. L., \& Newman, D. A. (2010). Emotional intelligence: An integrative meta-analysis and cascading model. American Psychological Association, 95(1), 54-78.

Kong, F. (2017). The validity of the Wong and law emotional intelligence scale in a Chinese sample: Tests of measurement invariance and latent mean differences across gender and age. Personality and Individual Differences, 116, 29-31.
Kökönyei, G., Urbán, R., Reinhardt, M., Józan, A., \& Demetrovics, Z. (2014). The difficulties in emotion regulation scale: Factor structure in chronic pain patients. Journal of Clinical Psychology, 70(6), 589600.

Kun, B., Balazs, H., Kapitany, M., Urban, R., \& Demetrovics, Z. (2010). Confirmation of the three-factor model of the assessing emotions scale (AES): Verification of the theoretical starting point. Behavior Research Methods, 42(2), 596-606.

Lang, P. J., Bradley, M. M., \& Cuthbert, B. N. (2005). International affective picture system (IAPS): Affective ratings of pictures and instruction manual. Technical report A-8. University of Florida, Gainesville, FL.

MacCann, C., Joseph, D. L., Newman, D. A., \& Roberts, R. D. (2014). Emotional intelligence is a second-stratum factor of intelligence: Evidence from hierarchical and bifactor models. Emotion, 14(2), 358-374.

Marchewka, A., Zurawski, Ł., Jednorog, K., \& Grabowska, A. (2014). The Nencki affective picture system (NAPS): Introduction to a novel, standardized, wide-range, high-quality, realistic picture database. Behavior Research Methods, 46(2), 596-610.

Mayer, J. D., \& Salovey, P. (1995). Emotional intelligence and the construction and regulation of feelings. Applied \& Preventive Psychology, 4, 197-208.

Mayer, J. D., Caruso, D. R., \& Salovey, P. (2016). The ability model of emotional intelligence: Principles and updates. Emotion Review, 8(4), 290-300.

Megías-Robles, A., Gutierrez-Cobo, M. J., Gomez-Leal, R., Cabello, R., Gross, J. J., \& Fernandez-Berrocal, P. (2019). Emotionally intelligent people reappraise rather than suppress their emotions. PLoS One, 14(8), e0220688.

Mikolajczak, M., Luminet, O., Leroy, C., \& Roy, E. (2007). Psychometric properties of the trait emotional intelligence questionnaire: Factor structure, reliability, construct, and incremental validity in a French-speaking population. Journal of Personality Assessment, 88(3), 338-353.

Mikolajczak, M., Petrides, K. V., Coumans, N., \& Luminet, O. (2009). The moderating effect of trait emotional intelligence on mood deterioration following laboratory-induced stress. International Journal of Clinical and Health Psychology, 9, 455-477.

Nielsen, L., \& Kaszniak, A. W. (2006). Awareness of subtle emotional feelings: A comparison of long-term meditators and nonmeditators. Emotion, 6(3), 392-405.

Pardeller, S., Frajo-Apor, B., Kemmler, G., \& Hofer, A. (2017). Emotional intelligence and cognitive abilities-associations and sex differences. Psychology, Health \& Medicine, 22(8), 1001-1010.

Peña-Sarrionandia, A., Mikolajczak, M., \& Gross, J. J. (2015). Integrating emotion regulation and emotional intelligence traditions: A meta-analysis. Frontiers in Psychology, 6(160), 1-27.

Petrides, K. V., Pérez-Gonzaález, J. C., \& Furnham, A. (2007). On the criterion and incremental validity of trait emotional intelligence. Cognition and Emotion, 21(1), 26-55.

Salovey, P., \& Mayer, J. D. (1990). Emotional intelligence. Imagination, Cognition and Personality, 9(3), 185-211.

Schutte, N. S., Malouff, J. M., Hall, L. E., Haggerty, D. J., Cooper, J. T., Golden, C. J., \& Dornheim, L. (1998). Development and validation of a measure of emotional intelligence. Personality and Individual Differences, 25(2), 167-177.

Schutte, N. S., Manes, R. R., \& Malouff, J. M. (2009). Antecedentfocused emotion regulation, response modulation and well-being. Current Psychology, 28, 21-31.

Shahzad, S., Begum, N., \& Khan, A. (2013). Understanding emotions in adolescents: Linkage of trait emotional intelligence with aggression. Asian journal of social sciences \& humanities, 2, 386-394.

Sheppes, G., Suri, G., \& Gross, J. J. (2015). Emotion regulation and psychology. Annual Review of Clinical Psychology, 11, 379-405. 
Smieja, M., Mrozowicz, M., \& Kobylinska, D. (2011). Emotional intelligence and emotion regulation strategies. Studia Psychologiczne (Psychological Studies), 49(5), 55-64.

Tamres, L. K., Janicki, D., \& Helgeson, V. S. (2002). Sex differences in coping behavior: A meta-analytic review and an examination of relative coping. Personality and Social Psychology Review, 6(1), $2-30$.

Thompson, R. J., Dizén, M., \& Berenbaum, H. (2009). The unique relations between emotional awareness and facets of affective instability. Journal of Research in Personality, 43(5), 875-879.

Vine, V., \& Aldao, A. (2014). Impaired emotional clarity and psychopathology: A transdiagnostic deficit with symptom-specific pathways through emotion regulation. Journal of Social and Clinical Psychology, 33, 319-342.
Whitman, D. S., Van Rooy, D. L., Viswesvaran, C., \& Kraus, E. (2009). Testing the second-order factor structure and measurement equivalence of the Wong and law emotional intelligence scale across gender and ethnicity. Educational and Psychological Measurement, 69(6), 1059-1074.

Zysberg, L., \& Raz, S. (2019). Emotional intelligence and emotion regulation in self-induced emotional states: Physiological evidence. Personality and Individual Differences, 139, 202-207.

Publisher's Note Springer Nature remains neutral with regard to jurisdictional claims in published maps and institutional affiliations. 